\title{
Barriers and facilitators to integrate oral health care for older adults in general (basic) care in East Netherlands. Part 1: Normative integration
}

\author{
Dominique Niesten $^{1}$ (D) | Anneloes E. Gerritsen ${ }^{1}$ | Verena Leve ${ }^{2}$
}

${ }^{1}$ College of Dental Sciences, Radboud University Medical Center, Nijmegen, The Netherlands

${ }^{2}$ Institut für Allgemeinmedizin, HeinrichHeine-Universität, Düsseldorf, Germany

\section{Correspondence}

Dominique Niesten, College of Dental Sciences, Radboud University Medical Center, P.O. Box 9101, 6500 HB Nijmegen, The Netherlands.

Email: dominique.niesten@radboudumc.nl

Funding information

Program: INTERREG V A Programms Deutschland-Nederland 2014-2020 (ANBest INTERREG DE-NL). Project name: Zorg Verbindt. Project nr 203072.

\begin{abstract}
Objective: to synthesise a framework of barriers and facilitators in the normative integration of oral health care $(\mathrm{OHC})$ into general health care for frail older adults at macro (system), meso (organisation and interprofessional integration) and micro (clinical practice) levels.

Background: Identification of these barriers and facilitators is expected to promote better and more appropriate care.

Methods: For this qualitative study, comprising 41 participants, representatives of 10 different groups of (professional) care providers, and $\mathrm{OHC}$ receivers (home-dwelling, and nursing home patients) in East Netherlands were interviewed. Transcripts of the in-depth, topic-guided interviews were thematically analysed. In a subsequent workshop with 52 stakeholders, results and interpretations were discussed and refined.

Results: Two main themes were identified: (1) a compartmentalised care culture in which $\mathrm{OHC}$ and general health care are seen as two separate realms, and (2) prioritisation, awareness and attitude regarding $\mathrm{OHC}$ integration. Subthemes such as low political attention (macro level); unclear responsibilities, hierarchical relations and the lack of vision of organisations (meso level); and poor awareness and low prioritisation by care providers and patients (micro level) were identified as potential barriers. Subthemes such as leadership (meso level), and the supportive personality of individual caregivers and ownership of patients (micro level) were identified as facilitators. Conclusion: Barriers and facilitators in normative $\mathrm{OHC}$ integration in The Netherlands are interrelated and apparent at macro-, meso- and micro levels. They are mainly related to (a) a compartmentalised care culture, and (b) related low prioritisation, and poor awareness of and attitude towards (integration of) oral health (care).
\end{abstract}

\section{KEYWORDS}

healthcare integration, interprofessional, normative integration, older people, oral health care 


\section{1 | INTRODUCTION}

Numerous studies worldwide have shown that oral health of older people, especially those with high or complex care needs, is generally poor, and professionally provided oral health care $(\mathrm{OHC})$ and oral self-care are inadequate. ${ }^{1-4}$ Reasons relate to personal and environmental factors such as (oral) healthcare organisation and policies. In order to improve the organisation of $\mathrm{OHC}$ for the group of older people with complex health needs, recent efforts have focused on exploring integrated care approaches, in particular the integration of $\mathrm{OHC}$ in primary healthcare practices. ${ }^{5-8}$

In a scoping review of 48 reports, Harnagea et $\mathrm{al}^{6}$ evaluated barriers to and facilitators of the integration of $\mathrm{OHC}$ in primary care in 18 countries. The barriers most commonly found were lack of political leadership and healthcare policies, implementation challenges, discipline-oriented education, lack of continuity of care and services and discrepancy between patients' and healthcare providers' perceptions of oral healthcare needs. ${ }^{9}$ The most commonly found facilitators were supportive policies and resource allocation, interdisciplinary education, collaborative practices between oral and other healthcare professionals, presence of local strategic leaders and geographical proximity of medical and $\mathrm{OHC}$ services. However, most of the healthcare strategies investigated focused on micro level processes (ie clinical services/patient-professional interactions) while evidence at the meso level (organisation and professional interaction) and especially at the macro level (system, politics) was relatively scarce and poor. Moreover, the majority of evidence related to the USA, Canada and Australia, with evidence from European countries almost exclusively derived from UK studies. Apart from a study on $\mathrm{OHC}$-related integrated care practices in three nursing homes in $2015,{ }^{10}$ no studies have focused on integrated $\mathrm{OHC}$ delivery in The Netherlands.

Following the World Health Organisation's (WHO) global strategy on people-centred and integrated health services, an evaluation of evidence on this topic concluded that "people-centred and integrated health services are essential components of building universal health coverage and can improve health status in countries of all income levels across the world" ${ }^{11}$ In order to design effective integrated care strategies, however, the local care delivery system and its relevant context must first be explored, since the ability to achieve successful outcomes through such strategies is highly context-specific. ${ }^{11}$ A second factor that has proven to contribute successfully to designing the effective organisation of integrated care is consultation of joint stakeholders in assessing needs, aims and conditions for integrated care practices (eg 11-13). A third vital element in the study of integrated care is the use of a conceptual model to frame results. ${ }^{14}$ An often used model is the Rainbow Model of Integrated Care ${ }^{15}$ (Figure 1). This model's simple and intuitive use has prompted researchers to apply it in several recent studies. The model distinguishes between care integration at four different levels: (1) macro: system integration; (2) meso: organisational integration; (3) meso: professional integration; and (4) micro: clinical service

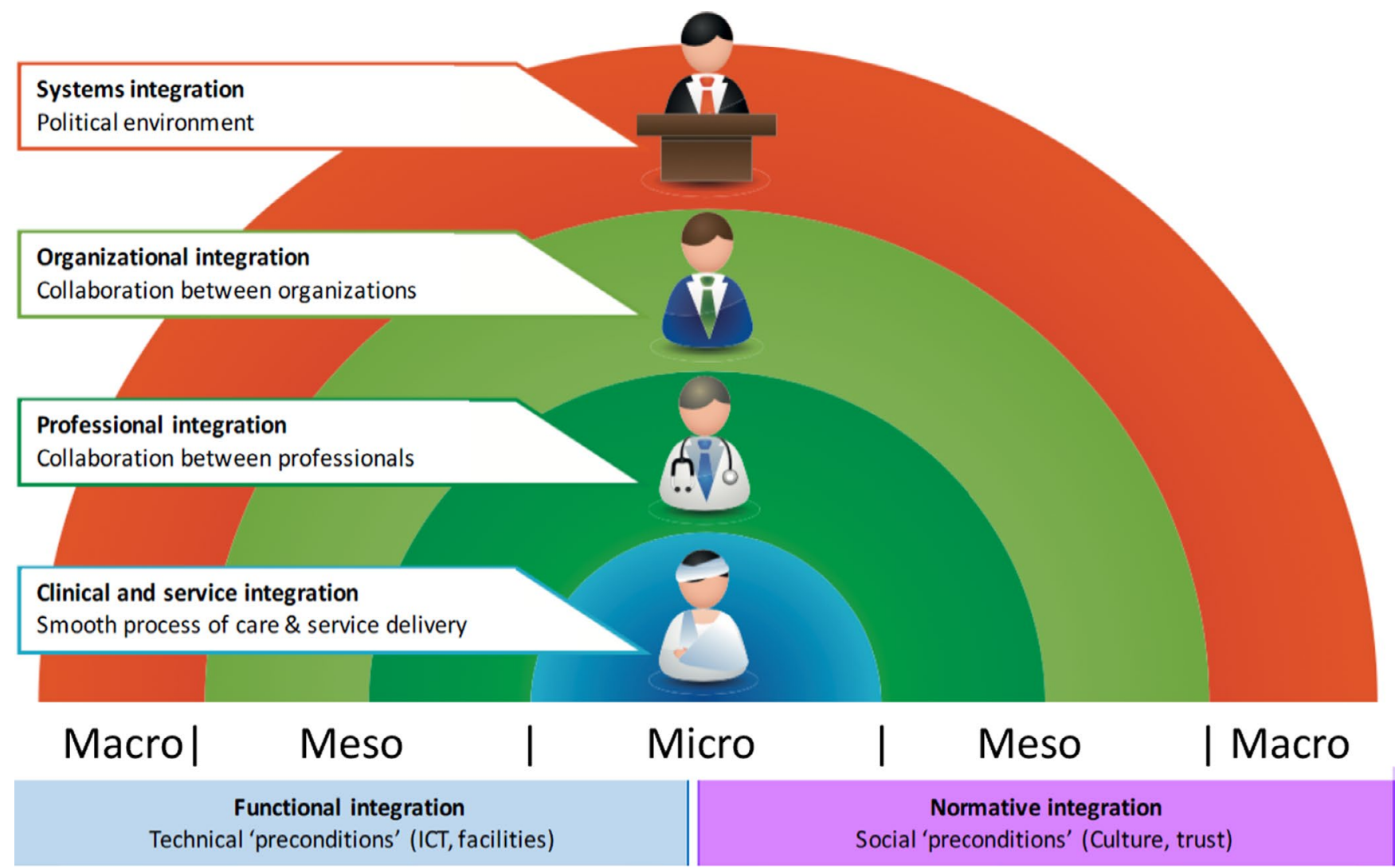

FIGURE 1 Rainbow model of integrated care (Valentijn et al, 2013 and 2016 ${ }^{15,52}$ ) [Colour figure can be viewed at wileyonlinelibrary.com] 
integration. It further distinguishes between functional and normative integration, both of which span and link all integration levels. "Functional integration" refers to functional aspects, that is, technical preconditions, and comprises mechanisms by which financing, information and management modalities are linked to bring optimum value to the system. "Normative integration" refers to normative aspects, that is, social preconditions, and comprises the development and maintenance of a common frame of reference (ie shared mission, vision, values and culture) between organisations, professional groups and individuals. ${ }^{15}$

The aim of the present study was to synthesise a framework regarding normative barriers and facilitators for the integration of $\mathrm{OHC}$ into primary (general) health care for institutionalised and home-dwelling (frail) older adults in East Netherlands (the Dutch side of the EUREGIO Rijn Waal ${ }^{16}$ ), distinguishing macro-, meso- and micro levels of health care.

The findings in relation to the functional aspects of integration are presented in a subsequent paper.

\section{2 | METHODS}

Since we aimed to study a relatively unexplored and complex topic, we carried out a qualitative study made up of individual interviews and a workshop.

\section{1 | Interview guide}

We (DN, AG, VL) developed an interview guide based on a scoping literature search on the integration of $\mathrm{OHC}$ into general or primary care, and on the Rainbow Model of Integrative Care. In the interviews, we first addressed the topic of facilitators of and barriers to $\mathrm{OHC}$ delivery in general, then we asked about experiences of and views on $\mathrm{OHC}$ integration, including any collaborative oral and general healthcare practices. We specifically asked what hampers or stimulates collaboration or integration of $\mathrm{OHC}$ in primary and nursing home care.

\section{2 | Interviews and workshop}

Respondents were selected purposefully through convenience and snowball sampling techniques, ${ }^{17}$ and through using personal networks and contacting care organisations active in a mid-east region of The Netherlands. Selection was geared to obtaining a 360-degree perspective of current OHC delivery, barriers and facilitators related to the implementation of integrated $\mathrm{OHC}$ in extra- and intramural settings. As such, 10 groups of stakeholders (dentists, dental hygienists, district nurses, nurses (nursing home), managers of (oral) care organisations, general practitioners (physicians), geriatric doctors, patients (home-dwelling and institutionalised) and informal caregivers in mid-east Netherlands) were targeted. Recruiting was stopped after two trained interviewers (DN, AG) had interviewed a minimum of three representatives of each group, and a certain degree of data saturation (no more than $5 \%$ new codes per interview) had been reached. All participants gave written informed consent. Interviews were transcribed verbatim and analysed.

After analysis of the interviews, a one-day "future" workshop ${ }^{18}$ was held among interviewees and additional stakeholders (policy makers in health legislation, health insurance and health education, and medical and dental students), in order to validate presented results and reach general agreement on outcomes (focus, themes and subthemes) and recommendations and thus help ensure their representativeness. ${ }^{18}$ During this workshop, we involved additional, purposively selected representatives of identified stakeholder groups. Interviews were held in 2017 and 2018; the workshop was held at end 2018. Detailed workshop minutes were checked by AG and DN for accuracy and then added to the database. In accordance with this information, themes and the coherence of identified themes and subthemes were verified.

\section{3 | Analysis}

We used a semi-directed approach to content analysis ${ }^{19}$ in order to enable and structure relationships between barriers and facilitators in $\mathrm{OHC}$ integration at different, predefined integration levels. Directed coding was used to categorise the data into two domains (normative and functional integration) and into three levels (macro, meso, micro) within each domain, based on the Rainbow Model of Integrated Care. ${ }^{15}$ Directed coding was also used to distinguish between barriers to and facilitators of integration of care. Emergent coding was used to identify themes within each domain and within and across levels. ${ }^{19}$ We used the definition of Grone and GarciaBarbero (2001): "integrated care is bringing together inputs, delivery, management and organisation of services related to diagnosis, treatment, care, rehabilitation and health promotion", ${ }^{20}$ to identify text segments on integrated care practices. Coding was conducted individually by DN and AG. Codes were cross-checked and deviating interpretations were discussed with a third researcher $(V L)$ until consensus was reached. A resulting list of themes, subthemes and findings was shared with interviewees, who were invited to participate in the workshop to validate results (member check). Coding, comparing and analysis of data were supported by the software program Atlas.ti8.

The study was approved by the Medical Ethics Committee (CMO) of the Radboud University, Nijmegen (CMO ref. 2016--3005).

\subsection{Role of the researchers}

Perspectives and knowledge related to various academic and professional backgrounds influenced the data analysis. The researchers added expertise in and knowledge of public $\mathrm{OHC}$ and philosophy 
(DN), public health and health sociology (VL), dentistry and dental geriatric care (AG), and qualitative methodology (all authors) to the analysis. During the analysis phase, we repeatedly consulted experts in the field (eg geriatric dentists, nurses and doctors) to discuss and explore the relevance of issues brought up during the interviews. We also revisited our observational notes, in order to generate contextual background for understanding and analysing the respondents' information.

\section{5 | Qualitative rigour}

Several techniques ensure the trustworthiness and credibility of our analysis. ${ }^{21}$ First, we triangulated information from three sources: interviews, observational notes and expert views, with the latter two serving to help interpret the information from the interviews. Secondly, we drew upon the multiple professional perspectives brought in by the respective backgrounds of the authors. Thirdly, we carried out member checks during the interviews (which involved restating or summarising information and then asking the respondents to determine its accuracy). Finally, the workshop served as a means of validating results, through asking participants involved to comment on the accuracy of initially derived themes and interpretations.

\section{RESULTS}

We interviewed 41 respondents from 10 stakeholder groups between May 2017 and June 2018, and discussed the results in a workshop of 52 participants in October 2018 (Table 1). A total of 5 dentists, 3 oral hygienists, 4 district (home) nurses, 4 nursing home nurses, 3 managers (two nursing homes and one dental carechain), 5 general practitioners (physicians), 6 specialists geriatric care (physicians), 7 patients ( 3 nursing home, 4 home dwelling) and 4 family caregivers participated. In the workshop, apart from the invited interviewees (14 out of 41 (34\%) attended), we additionally invited representatives from relevant groups that were not included in the interview phase. Among these were health policy advisors, insurance representatives, national health policy makers, dental teachers and students, and the dental curriculum director of Radboud University Medical Center (Nijmegen)-52 participants in total.

On the basis of the content analysis of interview transcripts, a preliminary list of normative barriers and facilitators in the integration of $\mathrm{OHC}$ into primary and nursing home care practices at macro-, meso- and micro level was presented to the participants of the workshop. The participants generally agreed with the presented results and interpretations; moreover, they helped to determine the importance, and thereby set the focus of presented themes and subthemes, as synthesised in Table 2. Most of the cited barriers were common to the participants of the workshop in both intra- and extramural settings. A selection of supporting quotes, derived from the
TABLE 1 Respondent characteristics (interviews)

\begin{tabular}{|c|c|c|c|}
\hline Stakeholders & Code & $\mathrm{F} / \mathrm{M}$ & $\mathrm{H} / \mathrm{N}$ \\
\hline \multirow[t]{5}{*}{ Dentist } & Dentist-H1 & $\mathrm{F}$ & $\mathrm{H}$ \\
\hline & Dentist-N1 & $\mathrm{F}$ & $\mathrm{N}$ \\
\hline & Dentist-HN1 & $M$ & $\mathrm{HN}$ \\
\hline & Dentist-H2 & $\mathrm{F}$ & $\mathrm{H}$ \\
\hline & Dentist-N2 & $M$ & $N$ \\
\hline \multirow[t]{3}{*}{ Oral hygienist } & OralHyg-HN1 & $\mathrm{F}$ & $\mathrm{HN}$ \\
\hline & OralHyg-N1 & $\mathrm{F}$ & $\mathrm{N}$ \\
\hline & OralHyg-N2 & $\mathrm{F}$ & $\mathrm{N}$ \\
\hline \multirow[t]{4}{*}{ Home nurse } & Nurse-H1 & $\mathrm{F}$ & $\mathrm{H}$ \\
\hline & Nurse-H2 & $\mathrm{F}$ & $\mathrm{H}$ \\
\hline & Nurse-H3 & M & $\mathrm{H}$ \\
\hline & Nurse-H4 & $\mathrm{F}$ & $\mathrm{H}$ \\
\hline \multirow{4}{*}{$\begin{array}{l}\text { Nurse (nursing } \\
\text { home) }\end{array}$} & Nurse-N1 & $\mathrm{F}$ & $\mathrm{N}$ \\
\hline & Nurse-N2 & $\mathrm{F}$ & $\mathrm{N}$ \\
\hline & Nurse-N3 & $\mathrm{F}$ & $\mathrm{N}$ \\
\hline & Nurse-N4 & $\mathrm{F}$ & $\mathrm{N}$ \\
\hline \multirow{4}{*}{$\begin{array}{l}\text { General practitioner } \\
\text { (GP) }\end{array}$} & Gen-Prac1 & $\mathrm{F}$ & $\mathrm{H}$ \\
\hline & Gen-Prac2 & $\mathrm{F}$ & $\mathrm{H}$ \\
\hline & Gen-Prac3 & M & $\mathrm{H}$ \\
\hline & Gen-Prac4 & $\mathrm{F}$ & $\mathrm{H}$ \\
\hline GP assistant & Gen-Prac-Ass1 & $\mathrm{F}$ & $\mathrm{H}$ \\
\hline \multirow{6}{*}{$\begin{array}{l}\text { Specialist Geriatric } \\
\text { Care }\end{array}$} & Spec-Ger1 & $M$ & $\mathrm{~N}$ \\
\hline & Spec-Ger2 & $\mathrm{F}$ & $\mathrm{N}$ \\
\hline & Spec-Ger3 & M & $\mathrm{N}$ \\
\hline & Spec-Ger4 & $\mathrm{F}$ & $\mathrm{N}$ \\
\hline & Spec-Ger5 & $M$ & $N$ \\
\hline & Spec-Ger6 & $\mathrm{F}$ & $N$ \\
\hline \multirow{4}{*}{$\begin{array}{l}\text { Home-dwelling } \\
\text { patient }\end{array}$} & Patient-H1 & $\mathrm{F}$ & $\mathrm{H}$ \\
\hline & Patient-H2 & $\mathrm{F}$ & $\mathrm{H}$ \\
\hline & Patient-H3 & $\mathrm{F}$ & $\mathrm{H}$ \\
\hline & Patient-H4 & $M$ & $\mathrm{H}$ \\
\hline \multirow{3}{*}{$\begin{array}{l}\text { Nursing home } \\
\text { patient }\end{array}$} & Patient-N1 & $\mathrm{F}$ & $N$ \\
\hline & Patient-N2 & $\mathrm{F}$ & $N$ \\
\hline & Patient-N3 & $M$ & $\mathrm{~N}$ \\
\hline \multirow[t]{3}{*}{ Manager } & Manager1 & $M$ & $\mathrm{~N}$ \\
\hline & Manager2 & $\mathrm{F}$ & $N$ \\
\hline & Manager3 & $M$ & $\mathrm{~N}$ \\
\hline \multirow[t]{4}{*}{ Family caregiver } & Fam-carer1 & $M$ & $N$ \\
\hline & Fam-carer2 & $\mathrm{F}$ & $\mathrm{H}$ \\
\hline & Fam-carer3 & $M$ & $\mathrm{HN}$ \\
\hline & Fam-carer4 & $\mathrm{F}$ & $\mathrm{H}$ \\
\hline
\end{tabular}

Abbreviations: $\mathrm{F}$, female; $\mathrm{H}$, home dwelling; $\mathrm{M}$, male; $\mathrm{N}$, nursing home.

interviews, that best illustrate the identified themes is presented in Table 3.

In the text below, themes and subthemes are indicated in bold print. 
TAB LE 2 Barriers (-) and Facilitators (+) to Normative Integration of Oral health care (OHC) in General care (GC). Main themes (first row) and subthemes (next rows) at macro-, meso- and microlevels

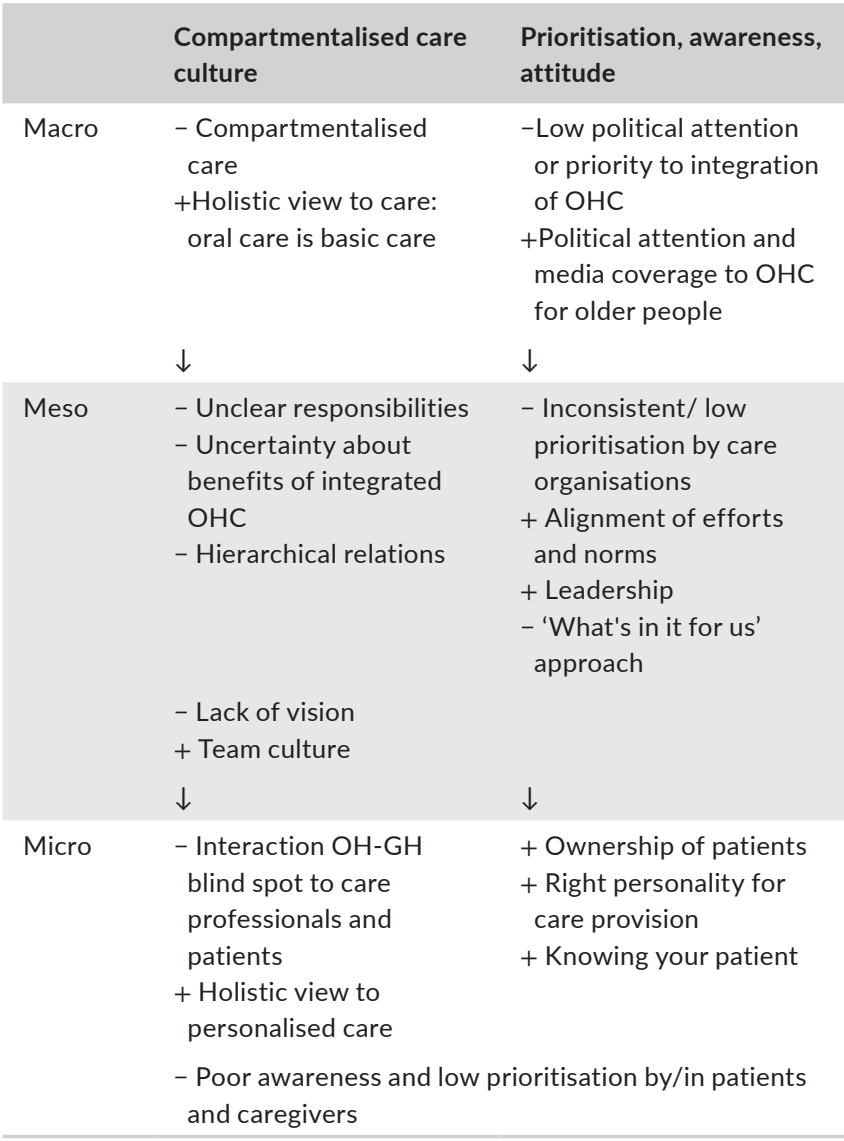

\section{1 | Theme: Compartmentalised care culture}

"Compartmentalised care culture" refers to the way that behaviour, norms, knowledge and beliefs (culture) of caregivers and care receivers are a reflection of the sharp division between dental and other care disciplines. This division itself, described as the subtheme compartmentalised care, was manifest at macro-, meso- and micro level and hampered integration of $\mathrm{OHC}$ into care (Q1, Q2). A compartmentalised care culture was encountered everywhere, starting at macro level. Policy makers did not conceive of oral care as part of basic care just like managers, care organisers, professional bodies and individual care providers at meso- and micro level. This was reflected in, and intrinsically linked to, the historically compartmentalised care system, where oral and general health care are separated in policies, financial arrangements (reimbursement and insurance schemes) and education.

The notion "oral care is so much more than just about those teeth" (Q3) was common among OHC providers; yet, the notion that general care was also about oral care was less apparent (Q4). The counterpart of the compartmentalised care culture was, not surprisingly, a holistic view to care, starting at system and policy (macro) level (Q5). Although generally not included in policies, visions or otherwise guiding formalised views at government or organisation level, several respondents expressed a holistic view of care.

At meso level, compartmentalised thinking led to unclear responsibilities in organisations where a certain level of $\mathrm{OHC}$ integration was implemented. In a nursing home where oral hygienists worked, nurses were asking: 'why should we put so much effort in cleaning the mouths when our patients see an oral hygienist every week?' About half of the interviewed non-dental care professionals, including nurses and GPs, agreed that they had a general responsibility for arranging or providing good $\mathrm{OHC}$, and for signalling common oral health disorders and informing dental professionals about these. However, the exact contours of these responsibilities were vague, as was the range of actions that this responsibility entailed (Q6). The question of whether bad oral health was caused by poor oral care or by a medical problem was important for apportioning responsibility; yet, at the same time, it was acknowledged that in practice this often cannot be determined as these factors are mutually dependent (Q7).

Uncertainty about benefits of care integration or even collaboration was another result of compartmentalisation. Some of the interviewed GPs and home nurses were unsure whether more collaboration with dentists or more dental knowledge would improve the care they delivered (Q8-Q10). This was different for most specialists in aged care (physicians who work in nursing homes) and nurses in nursing homes, who recognised the need for cooperation more clearly. Strikingly, most home nurses and GPs said that they did not encounter many oral health problems in their patients, but also admitted that they never (nurses) or only rarely (GPs) performed oral examinations and that their skills and knowledge related to diagnosing oral disorders were very limited. "I don't see it so it doesn't exist" was the underlying common circular reasoning.

Apart from "horizontal" compartmentalisation (ie different care disciplines like ergotherapy and oral care operating in separate care domains), we were also informed about "vertical" care compartmentalisation, mostly caused by hierarchical relations between care providers working at different organisational levels. Such hierarchy complicated $\mathrm{OHC}$ integration through restricted communication in daily care and existed, for instance, between nurses versus oral hygienists, and oral hygienists versus medical doctors and dentists, but also between other levels of care, for example, family caregivers versus nurses and among nurses of different education levels (eg Q11, Q12). In general, cooperation between caregivers of different levels was good, but this required good communication skills, especially of those "higher" in the hierarchy (Q13).

At micro level, a compartmentalisation culture meant that the interaction between oral health and general health was a blind spot for most care professionals and patients. This meant that most patients would not ask for daily $\mathrm{OHC}$ support or raise the topic of $\mathrm{OH}$ to care professionals other than $\mathrm{OHC}$ professionals, perceiving $\mathrm{OH}$ and general health areas as separate realms. Especially in cases of cognitively impaired patients with health problems, most care professionals would not be alert to possible oral causes of these health problems (Q14). General practitioners would not offer $\mathrm{OH}$-related services, or easily signal $\mathrm{OH}$ disorders when patients would present 
TABLE 3 Quotes of interviewees $(n=41)$ supporting identified themes and subthemes

\section{Compartmentalised care culture}

Q1 Oral care, that is not always seen as basic care. Not by everyone, not at all. It is not conceived that way [Nurse-N4]

Q2 There is a culture of pigeonholing in care. Oral care is one pigeonhole, it's not seen as part of a whole. [Fam-carer1]s

Q3 ...Why shouldn't you make that connection [between nurses and oral hygienists] so that they learn very specifically, how should I do that with older adults or how should I deal with this or that. Because oral care is so much more than just about those teeth. [OralHyg-N1]

Q4 The profession of dentist is about as far from me as that of a vet. [Gen_Prac2]

Q5 I think that oral care is really just basic care. So you don't have to make it special. [...] the organisation itself should actually perform an audit every time where all aspects of personal care would be included, including oral care. Because then, yes, then it becomes much more of a whole. [Spec-Ger1]

Q6 It is very difficult to say, if you are responsible for oral care, to what extent that is. And how do I bring that responsibility into practice? How do I do that? [Spec-Ger2]

Q7 if a concern is purely a care problem, then it should lie with the care team. And you may wonder if dental issues are rooted in medical or in care problems. Thing is that when care, or self-care, is poor, then medical problems develop. [Spec-Ger6]

Q8 I don't know how a dentist could help us. [Gen-Prac2]

Q9 Also when it comes to oral care, they often think: we have to do something extra again. [...]. So what do we gain by it? What interest does the professional have at that moment to do it. That is a very good question. To be honest, I would not immediately know the answer. [Nurse-H1]

Q10 [in answer to the question if GPs would be prepared to gain more knowledge on $\mathrm{OH}$ ]: There must be benefits for the doctor. This may involve financial compensation for training, or the doctor must be convinced that knowledge about oral health really leads to better care provision. [Gen-Prac3]

Q11 I personally find the contacts between us and the doctors much less transparent and much more difficult. [...] of course I do fewer things that are invasive. And then in the MDO [multidisciplinary consultation], among specialists, I feel "I'm only the oral hygienist" [...] and dare not say everything. [OralHyg-HN1]

Q12 As a [family] caregiver you have to be careful, they think you know nothing. The nurse always knows better. Like she insists on handling the wheelchair lift: "Let me do this." As if I don't know. And sometimes I say to her, well, I handle it the whole day through, but that doesn't impress her. Haha. [Fam-carer2].

Q13 Never say to the nurses "that's really not good," "That still has to be done." Then their hairs stand right up and they dig their heels in. [...] you have to keep a good relationship with the care worker, so I rather say: "that's really good. You did a good job. Could you then perhaps pay a little more attention to this?" [OralHyg-N2].

Q14 If you inspect the mouth, you can just see the big things. But I think it is almost more important, for example, people who just have general ill-feeling complaints, not feeling well, lack of energy. [...] especially then we should think about the mouth. If they lose weight, yes that could be end-stage dementia. But please beware, their mouth might hurt. They can't eat anymore. And a person with dementia cannot indicate it clearly, like saying "it hurts here," as a doctor you will have to be alert. [...] It will not be the first time that rotting retained roots cause those health complaints. [Spec-Ger5]

Q15 there was a man with various brain tumours, and he always had a sub-febrile temperature, like $37.5,38,38.2^{\circ} \mathrm{C}[\ldots]$. And that went on for a year. [...] So at a certain point we decided to lift that very vulnerable man out of bed in a wheelchair and move him to a dentist, who extracted his teeth, removed everything. [....] from that moment he was fine. [...] But later, doctors with whom I later discussed similar cases, they have contested that. "No, that is not possible, because his general health and functioning could not have been troubled by this mouth problem." [Spec-Ger2]

Q16 What I sometimes do is mention the relationship between health and oral health. That at some point I say it could be bad for your heart or something, then they will start to listen. [...] Like, "you're a heart patient and it seems better if you...." [Dentist-HN1]

Prioritisation, awareness, attitude

Q17 I think that we [dental personnel] and the caregivers have to head the same way, especially since they are most closely involved with the residents. [...] if the manager says, well, oral health care does not have priority or something, then I can imagine.... There must be a kind of tail wind in all organisation layers, otherwise you cannot bring them together. But that is difficult indeed. How do you get them [the different care providers] together? Education I think, and clear communication on what you want across all those different layers. [Dentist-N2]

Q18 Management, that also includes ongoing shifts, changing it again every 3-4 years, renewing it again and having new ideas and money is the biggest problem. [...]. Then there are priorities and we [dentists/ oral healthcare providers] have the least priority. [OralHyg-N2]

Q19 Intramurally, in the nursing home, it [OHC] has been given a certain priority, because we are obliged to include it in the patient's care plan. It is not like that in the extramural situation yet. When I am in an MDO [multidisciplinary consultation] and discuss a care plan with a GP, he looks at me in a very strange way when I start talking about oral care. [Spec-Ger5]

Q20 [poor oral health of older population] caught the attention of the Ministry of Health, Welfare and Sport and that led to stricter inspections by the IGZ (Ministerial Health Care Inspectorate). In 2013 the IGZ visited 29 nursing homes; 27 of them did not meet the standards for good oral care. Fortunately, a lot has changed since then. There are no longer any nursing homes in the country that have no formalised cooperation with a dentist; before 2013, that was rare. [Manager2]. 
TABLE 3 (Continued)

Q21 It [importance of good $\mathrm{OHC}$ ] was also in the news a while ago, like poor oral care increases risk of pneumonia etcetera. That was on nu.nl (popular Dutch news app). Then they knew about it in the MDO [multidisciplinary consultation], and it got extra attention and gained importance, like "we have to do something about it". [Spec_Ger6]

Q22 Simply promoting the importance of good oral care, so that older adults themselves are convinced of that and ask for the care they need [...]. So some form of national education would help, yes, you used to have these national health promotion adverts on TV. [Nurse-N1]

Q23 So that guideline was a good reason to put dental care higher on the agenda. In any case, to pay more attention to it, in the sense that we all thought okay, we do need good oral care. [Spec-Ger3]

Q24 No, but I will not do that [visit a dentist because or ill-fitting prostheses [...] No, but that's not worth the effort for me anymore. It is too trivial for that. [Patient_H3]

Q25 No, I suffer from rheumatism, it is so much pain, then I don't feel like going to the dentist anymore. No, I say, I will let that go. [...]. And I brush less often.[...] I can't bear it anymore. [Patient_N1]

Q26 It is often the nurses who find it very important themselves and who visit the dentist or dental hygienist themselves every 4 mo or every six months. And those who do not do that themselves, you notice that they provide less frequent $\mathrm{OHC}$ to the residents. [OralHyg-N1]

Q27 I think that there are still a lot of GPs, and that is all very understandable, with time constraints and everything, are just very reactive. [...]. If they hear about dental problems, already on the phone they say "go to the dentist first". And I think there are few GPs who actively monitor weight, malnutrition. I don't even do that myself. [Gen-Prac4]

Q28 I don't know how the oral health and oral hygiene of my patients are, I never ask. [Gen-Prac2]

Q29 Knowing that you have to do it does not mean that it is done well. It also involves behavioural change. [...] So as long as nobody is paying attention, it won't happen. And that's why I always say that the caregiver must do it, but the manager makes the difference [...]. If the manager says: in my department, or my house, or my organisation, oral care is important, and I see to it, and I make sure that you are facilitated [...] then you get better care. [Spec-Ger1]

Q30 Good oral healthcare implementation happens [...] because I feel responsible, because I am the one in the organisation who, after all, has a bit of a pioneering role. Or pull the cart. That is also part of my motivation. [Nurse-N3]

Q31 [in answer to a question about the effects of $\mathrm{OH}$ training] That depends very much on the attitude the oral health champion has. If you have an active oral health champion, he or she will be very active with that theme and will retrieve much more information and will be much more accessible for colleagues than someone who takes a low profile. [...] you see where the OH champions are a bit more active, that they can inspire and stimulate the colleagues very well, [...] for example, when they are present when the doctor visits the patient, they often ask questions like "how is the oral care, the oral health?"' [...] "How have things gone in the past few weeks?" Then they get people to start talking about oral care. [Nurse-N1]

Q32 It has to do with your aura, it has to do with your attitude, it has to do with customer friendliness. I think that many people are focused on ADL [activities of daily life], but not on the person. Psychological well-being has nothing to do with ADL care or good food. That has to do with being seen, heard and appreciated as a human being. That you really work in a customer-friendly way, [...]. When you help someone through ADL care, then you should know that that person is ashamed. Then you need to know how that you really infringe on someone's privacy [....] That requires some other competencies than just being able to give good ADL. [Manager3]

Q33 Well, after asking if I could see her self-embroidered paintings, she finally let me look into her mouth. Otherwise she won't open her mouth. But therefore you have to know your people. And then knowing what works for this patient, what doesn't work, and what works for that patient [...] That is even more important than knowledge about oral care. [OralHyg-HN1]

Q34 Yes, you must also be resourceful. And be persuasive. And sing songs. I sing songs with them. I can't sing at all, but I do sing songs. And then that mouth opens. And then I sing... aaaah... and song with a lot of "aaaaaaaaaaaaaaa". And -whop- insert a mouth mirror [ instrument]. [OralHyg-HN1]

Q35 I also know this woman who said "if you ever knew how meticulous my husband was about his teeth. And then look how it is right now. It's a good thing he doesn't know anymore. Because he would have found it terrible. [...] He flossed and he used those interdental cleaners, he cleaned and polished and you should see what it looks like now." And then I think, I am 100\% committed to at least keep this mouth clean and free of pain, those last months he has left. But of course I can't do that on my own either. But if we [oral hygienists] see someone come more often, the department thinks "oh, he frequents the oral hygienist", so we should do our best a bit more. [OralHyg-N2]

Q36 I find it very difficult to motivate certain people, like change their health behaviour. I have come to be a bit fatalistic about that. When you see overweighted people who smoke and use alcohol and whatever.... who don't brush either and whom I send to the dental hygienist every six months... I really don't have the feeling that I can change their behaviour through motivational interviewing or such things. I have lost my idealism, that I would change the world someday. I can't. [Dentist-HN1]

Q37 Another point is if the caregivers do it [provide $\mathrm{OHC}$ ], how do they know if they're doing it right and effectively? You know, caregivers think that if they don't brush they save time. So they're done earlier. They say "The clients, if they ask for it, I'll do it. But if I do it quickly, or if I say well I'll do it tonight, really no one would care". [...] Caregivers don't see patients' health improve when they brush, at best these patients say "thank you for brushing my teeth, because it's nice and fresh now". But whether they finally receive better care because of it, or whether or not they get healthier more quickly, caregivers don't see that. [Spec-Ger1] 
TABLE 3 (Continued)

Q38 I think the threshold to ask for advice from an expert or to introduce a new idea, that threshold is relatively high or sometimes district nurses don't think about it at all. Or the need has to be very high. They're used to solve problems themselves, sort it out. It's culture [...] Perhaps the idea is: I should know this, because as a general nurse you have to know a lot. And during staff trainings, that is not always nice, I must say. That too is culture. Pointing the finger, saying; "you're not doing it right". Nurses who train nurses do that very often. That is not a good climate for learning. [Nurse-H1]

Q39 Culture varies a lot, also from unit to unit. Some units do their very best. It only takes one rotten apple to ruin it and then tyrannise other units. And then nothing happens, then really nothing happens. Then you try with all your might try to turn that ship somewhat, then when it is turned 1 or 2 degrees, that ship, you should be very happy. And if you don't pay attention, then it turns back again. They are stuck in their habits. "we have done it like that for years, and it has always gone well and now you come to tell us how we should do it". [...]. [OralHyg-N2]

Q40 I am afraid that oral care is very much about money. [...] I think that many dentists are geared towards providing technical state-of-the-art solutions. And making money. And that is pre-eminently something you should not do in providing oral health care for older adults. You first have to look at the people and their needs and perceptions. [...] The aim of a number of dentists is to enrich themselves, not primarily to care about the interests of patients. [Dent-HN1]

Q41 You see that those [dental] organisations note that the oral hygiene is poor and then they take over oral cleaning from the [nursing] home. They bring in a few prevention assistants. They arrange a cleaning team that goes in every week or every two weeks to clean the elders' teeth. But that is not how it should be, the carers are supposed to do that. And then they might say, "we can't tolerate that the oral cleaning is so poor here" [...] but most of them do it to deploy those prevention assistants at a dentists' hourly rate [Dent-N1]

with general health complaints that could be related to $\mathrm{OH}$ (Q15), apart from, sometimes, malnutrition-related issues. Most specialists in aged care and nurses, on the other hand, demonstrated awareness of the possible relationship between physical and behavioural disorders and oral problems. OHC professionals used the relationship $\mathrm{OH}-\mathrm{GH}$ to motivate unknowing patients to improve their oral hygiene (Q16).

\section{2 | Theme: Prioritisation, awareness, attitude}

The second main theme referred to the level of importance given to the integration of $\mathrm{OHC}$ into general health care, as well as the awareness of $\mathrm{OH}$ (care) implications and the attitude towards $\mathrm{OHC}$ provision.

In general, respondents agreed that there was no or low political attention or priority to integration of $\mathrm{OHC}$ into general care among policy makers, care managers and care professionals alike. $\mathrm{OHC}$ received low priority in general, especially in the home-care situation, and this was reflected in a lack of vision regarding $\mathrm{OHC}$, or an inconsistent vision. Yet, respondents, both care providers and managers, agreed on the importance of a clear vision and policy, consistence and continuity in priorities, and the need to align the efforts to this policy throughout the organisation (Q17). A clear vision for care that includes $\mathrm{OHC}$ was, however, only reported by representatives of one nursing home; all other interviewees reported lacking this vision. A major reason for this deficiency, and for inconsistent and low prioritisation by care organisations at meso level, was the absence of a holistic care view: oral care is general care (see Q5). Lack of (long-term) vision was also furthered by the ongoing changes in political leaders, national priorities and policies (macro level), as well as by high rates of management and care-staff turnover at meso- and micro level (Q18). Nevertheless, integrating $\mathrm{OHC}$ into general care practices still took a certain priority in nursing homes, and priority to and awareness of the importance of $\mathrm{OHC}$ were clearly higher in nursing homes than in the homecare situation (Q19). This was mostly because of enforcement of the implementation of the Oral Health Care Guideline in nursing homes, which in most homes had occurred in the past 5 to 10 years (Q20), and because of national media coverage, for instance on the poor oral health of nursing home residents. This recent political attention to and media coverage of OHC for older people had, according to managers and care providers, put $\mathrm{OHC}$ on the agenda of care institutions (Q21). National media coverage not only helped to put $\mathrm{OHC}$ higher on the political agenda; it also served as a promotion tool for increasing awareness and good oral health behaviour among care providers and patients. Besides, it helped to match $\mathrm{OHC}$ demands to $\mathrm{OH}$ needs among frail older people (Q22). It also contributed to alignment of efforts and norms among care providers, just as the $\mathrm{OHC}$ guideline (see 22) and quality auditing that used this (non-compulsory) guideline as a base for monitoring quality of $\mathrm{OHC}$ had helped to align norms with regard to the necessity of good oral care in nursing homes (Q23). At the same time, it was acknowledged that political attention mostly comes in waves and that changing political priorities, as mentioned above, and the resulting changing focus of Inspection boards and care institutions' policies jeopardise the continuity of care, as do the shortage and high turnover of staff.

At micro level, frail older patients generally showed poor awareness of the benefits of $\mathrm{OHC}$ and some had an unfavourable attitude towards dental visits or oral self-care provision (Q24, Q25). Poor awareness in and low prioritisation by patients and care providers was present, for instance, in nurses with lower-level training and care providers and patients who had poor OHC habits (Q26). OHC also had low priority for most GPs, whose lack of awareness and lack of interest in oral health and $\mathrm{OHC}$ were often mentioned barriers to $\mathrm{OHC}$ integration in primary care, even by GPs themselves (Q27, Q28).

For $\mathrm{OHC}$ integration at meso (organisations) level, but also for adequate $\mathrm{OHC}$ delivery in general, managers played a major role. 
Leadership-referring to the ability of a person or organisation to lead or guide other people, teams or organisations-was deemed to be crucial (Q29). Leadership of managers and team leaders, but also "personal" leadership of caregivers, could make the difference between adequate or poor (or no) $\mathrm{OHC}$ integration.

Personal leadership, in particular exerting responsibility, was another facilitator that was recognised by nurses. This related to doing what needs to be done to initiate or maintain good (oral) care practices and taking initiative because one feels responsible and intrinsically motivated to do so (Q30). This played a role at all levels of care. In the absence of structures for the delivery of integrated care, good practices were often fully dependent on individual people. These could be nurses, but also family caregivers, geriatric doctors and managers. One way to increase ownership was to appoint "oral health champions," which was achieved in about half of the nursing homes. "Oral health champions" were responsible for stimulating and supporting adequate implementation of daily $\mathrm{OHC}$, for example by organising $\mathrm{OHC}$ training of other nurses. There was a large difference between organisations and wards where such $\mathrm{OH}$ champions were self-motivated and active and those where they were appointed despite their lack of interest in $\mathrm{OH}$. In the first case, $\mathrm{OH}$ champions could help evoke responsibility and motivation in nurse colleagues and generate awareness of $\mathrm{OHC}$ as a standard issue in care evaluation (Q31). Creating a sense of responsibility among unmotivated caregivers was a big hurdle. However, it was felt that the right person could motivate even less enthusiastic colleagues, and that results of an active attitude could be seen in the entire team and in the oral hygiene status of residents.

A key facilitator was availability of caregivers with the right attitude, the right personality for providing personalised care. At meso level, it was important, but very difficult, to attract such caregivers. "Knowing your patient" and emphatically acting upon that knowledge were essential for providing requested personalised care (Q32), including $\mathrm{OHC}$, in a way and at moments that patients were open to receiving it. Knowing your patient was, according to some caregivers, more important than $\mathrm{OHC}$ knowledge and skills (Q33) and deemed to be a crucial facilitator for delivery of integrated $\mathrm{OHC}$ Other aspects of the "right personality," especially with cognitively impaired patients, were patience and resourcefulness. Some OHC providers were highly creative in overcoming their patients' resistance to opening their mouths (Q34). Another vital aspect of the "right personality" was motivation, a factor that was also mentioned by and about patients. Ownership of patients related to patients taking responsibility for their own oral health through taking the initiative to arrange their own $\mathrm{OHC}$, for example, through asking for support with OHC or for dental visits. Such ownership, according to oral care professionals, family caregivers and nurses, made substantial differences to the efforts they would make to (help) provide good OHC (Q35). Patients who demonstrated ownership through asking for oral care or expressing interest in good oral health not only received better and more consistent care, but also helped to increase awareness among care providers. On the other hand, oral care providers complained that it was difficult and often nearly impossible to motivate uninterested patients and their family caregivers (Q36). It was said that, while results of good wound care were obvious when wounds started healing, results of good $\mathrm{OHC}$ were less recognisable. This sometimes gave rise to a "who cares if I do that or not" attitude (Q37).

Team culture, which relates to the values, beliefs, attitudes and behaviours shared by a team, was identified as a barrier as well as a facilitator at meso level. Especially the culture within a nurse-team, either intra- or extramural, was essential for the integration of $\mathrm{OHC}$ into care procedures. Nurses and dental professionals experienced large differences between team cultures, especially with respect to learning climate, ways of relating to and interacting with colleagues and other caregivers, and communication. These differences were seen between and within organisations. Especially in home care, respondents indicated that there was no good climate for learning and that nurses were inclined to give negative rather than constructive peer feedback (Q38). Resistance to new care practices was also common in nursing home wards (Q39). Culture was in part modifiable by good management (eg Q30). Having to hire unmotivated or lower-level care personnel, which happened regularly owing to workforce shortage, generally jeopardised a positive team culture.

Finally, at meso level, a "what's in it for us" attitude was identified as a barrier. By some respondents, mainly $\mathrm{OHC}$ professionals, dentists were blamed for allowing financial benefit to prevail over care benefit (Q40). Financial incentives seemed to trigger certain dentists (or dental organisations) to focus on generating revenue (Q41) above investing in, less lucrative, collaborative care.

\section{4 | DISCUSSION}

This study explored the barriers to and facilitators of normative integration of $\mathrm{OHC}$ in general care in East Netherlands. Overarching barriers were a compartmentalisation culture and low priority and attention given to integrative care practices. Barriers identified were to a large extent interrelated and in some cases showed a degree of overlap. They will, therefore, have to be tackled with regard to their interdependence and across macro-, meso- and micro levels.

This study has some limitations. First, not all stakeholder groups were included in the interviews. We had to limit the number of stakeholder groups to enable both the manageability of data and a minimum degree of data saturation through setting a minimum to the numbers of interviewees per stakeholder group. ${ }^{23}$ Hence, we did not interview-for example-representatives of speech therapists, dieticians and ergo therapists, and policy makers were included only in the workshop. Second, numbers of interviewees within stakeholder groups were low (three to five), and we therefore might have missed views or attitudes. This limitation was partially mitigated by the invitation of additional participants to the workshop. Third, for funding reasons, we had to restrict our research to a mid-east region of The Netherlands. Nevertheless, when checked in national workshops and presentations implemented as part of the Interreg "Zorg 
Verbindt" project, found results appeared to be representative for The Netherlands at large, with the exception of some identified best practices at meso- and micro level.

A strength of the study is that, through the inclusion of a large variety of stakeholders at macro-, meso- and micro levels of care, we could uncover and link barriers and facilitators across these levels, and hence obtain a comprehensive view of the topic. Another methodological asset is the workshop for stakeholders, which gave ample opportunity for collecting additional context and nuance, verifying our findings and extending these to represent more stakeholders.

In this study, the first overarching barrier to normative integration of $\mathrm{OHC}$ into basic (general) care practices was a compartmentalisation culture, manifest at macro-, meso- and micro level. The fact that $\mathrm{OHC}$ has been separated historically from general health care (systems), both clinically and administratively, has been stressed by several authors. ${ }^{7,8,24}$ Yet, as long as $\mathrm{OH}$ is conceived of as something separated from and prioritised lower than overall health by policy makers, managers and care providers alike, we are a long way off from organising and delivering integrated OHC. In terms of normative integration (the establishment and maintenance of a common culture and norms across units and organisations within a health system ${ }^{25}$ ), a common culture and shared norms were found to some extent only among specialists in aged care and $\mathrm{OHC}$ professionals engaged in or in favour of collaborative health practices with the aim of improving oral health, without doubting the benefits and the value of the efforts required. On the other hand, other care providers, including GPs, nurses and family caregivers, were mostly uncertain about the benefits (outcomes) of $\mathrm{OHC}$ after integration and unclear about their responsibilities regarding $\mathrm{OHC}$ provision. Similar findings were reported in a systematic review on barriers to $\mathrm{OHC}$ provision, which showed that the role of "beliefs about the importance and beneficial consequences of $\mathrm{OH} / \mathrm{OHC}$ " varied widely among caregivers, and that "unclear responsibilities for oral care" were seen as a major barrier. ${ }^{26}$

Yet, shared norms, clear and common goals, and clear professional roles and responsibilities are considered crucial to the commitment of caregivers in providing integrated or collaborative care (eg 27,28). Commitment, in turn, is linked to people's intention and behaviour and, as such, is a main predictor of the effectiveness of (integrated) health care. ${ }^{28}$ Uncertainty regarding the benefits of integration of $\mathrm{OHC}$ outcomes seems a logical result of the fact that most participants had no concrete idea of what integration could or should entail. Research on effects of interprofessional collaboration within health care is still in its infancy and evidence for the effectiveness of this type of collaboration is scarce and weak. ${ }^{29}$ This is even more so in $\mathrm{OHC}$, where best practices are largely unknown, ${ }^{30,31}$ as was shown in our study. Yet, belief in potential positive effects of care integration is a vital element of effective interprofessional collaboration. ${ }^{32}$

The subtheme hierarchical relations between care providers as found in the present study was also documented in a systematic review on $\mathrm{OHC}$ provision. ${ }^{26}$ Likewise, power distance (a consequence of role-related hierarchy) has been recorded as a barrier to effective interprofessional collaboration. ${ }^{33-35}$ Moreover, evidence exists for the usefulness of a mediation model that predicts that, as perceived power distance decreases among collaborating individuals, psychological safety (the belief that one can express oneself without fear of negative consequences), perceived team cohesion and perceived team effectiveness increase. ${ }^{33}$ Hence, in order to achieve more effective collaboration, it may be helpful to decrease power distance, for example, through joint training or interprofessional education for caregivers of different levels and professions. ${ }^{33}$

The second overarching theme was prioritisation, awareness and attitude. The reported subtheme lack of priority of integrating $\mathrm{OHC}$, although not in line with the steadily increasing attention paid to interprofessional collaboration in health care over the last decades since the WHO's calls in 1988 and 2010, ${ }^{36,37}$ is reflected in the small numbers of reported structural $\mathrm{OHC}$ integration practices worldwide. ${ }^{5,38,39}$ Unsurprisingly, visions of $\mathrm{HC}$ delivery that incorporate $\mathrm{OHC}$ are virtually non-existent. Yet, a clear vision that is grounded in evidence, together with political leadership that prioritises integrative (oral) health care, and evaluates improvements based on data, is considered necessary for sustainable (oral) healthcare implementation. ${ }^{40}$

Although leadership with regard to $\mathrm{OHC}$ integration was not identified at the macro level (politicians), we did come across several examples of leadership at meso- (managers and team leaders) and micro levels (care providers and patients). Leaders shared their vision, empowered others, and took initiatives to organise $\mathrm{OHC}$ within existing care practices. These initiatives were a major drive for $\mathrm{OHC}$ integration not only through alignment of norms (the norm being that $\mathrm{OHC}$ is an integral part of general basic health care), but also through creating purpose and direction, interest in and an understanding of each other's tasks, and a (more) positive team culture. The initiatives also involved the establishment of clear(er) tasks and responsibilities, for instance through the use of the Verenso OHC guideline $^{22}$ or through the appointment of "OH champions". Such interventions are known to work well only if supported by adequate leadership. ${ }^{41}$ Investing in leadership, that is, appointing personnel with leadership-related personal qualities (eg self-belief, drive for improvement, ability to empower others ${ }^{42}$ ), is likely to help improve $\mathrm{OHC}$ integration.

In addition to leadership, our findings stressed other subthemes, such as having the right attitude and personal qualities for providing integrated $\mathrm{OHC}$. Care providers, especially nurses, who showed patience, resourcefulness and empathy; had good communication skills; and knew their patients were considered to provide better $\mathrm{OHC}$ and to facilitate better $\mathrm{OHC}$ integration through better cooperation with other caregivers. Patients refusing $\mathrm{OHC}$, a barrier that was often mentioned by formal caregivers and managers in a systematic review, ${ }^{26}$ was rarely or not reported by informal or family caregivers. This might indicate that the setting and, especially, the relationship between the dependent older person and the caregiver are more important for providing care to patients that refuse care than is training in $\mathrm{OHC},{ }^{26}$ as also shown by Garrido et al. ${ }^{43}$ As several nurses and managers in our study reported, it is difficult to attract 
motivated personnel with adequate personal qualities. This is likely to become a definite problem as a demand for 40 million additional health workers is expected globally by $2030 .^{44}$

Influencing norms with regard to integrated care practices becomes difficult if health workers show little intrinsic motivation for cooperation or extending their care practices. Shared norms are, however, vital to the establishment of shared goals, mutual trust and effective communication, which in turn are essential for effective integrated $\mathrm{OHC}$ practices. ${ }^{45}$

Shared norms can be established by engaged care professionals, but also by patients as co-creators of their oral health, for which they need to take ownership, as expressed by some respondents in this study. The concept of patient ownership has been connected to commitment, responsibility, accountability, advocacy and continuity of care ${ }^{3,15}$ and can be provoked by citizen empowerment, which might be an important key to $\mathrm{OHC}$ improvement. ${ }^{40,46}$ Such empowerment would also increase the low level of priority given to $\mathrm{OHC}$ by many patients and certain care providers. However, when dealing with frail older people, many of whom have cognitive impairments, empowerment would have to begin at a pre-frail age.

Finally, the "what's in it for me" attitude of oral health providers is reflected in recent health insurance figures on costs declared by $\mathrm{OHC}$ providers in homes for older people. These have risen substantially, for example, by over 25\% between 2017 and 2018. This rise is substantially higher than that within any other health category and cannot be explained by increased oral health needs or higher numbers of patients. ${ }^{47}$ From the literature, it is clear that, in creating optimal conditions for cost-efficient integrated $\mathrm{OHC}$ practices, the financial motivation, being related to remuneration schemes of $\mathrm{OHC}$ providers, needs particular attention. ${ }^{48-51}$

\section{5 | CONCLUSION}

Barriers to and facilitators of normative integration of $\mathrm{OHC}$ in The Netherlands are mainly related to (a) a compartmentalised care culture in which $\mathrm{OHC}$ and basic (general) health care are seen as two separate realms, and caregivers and patients are insufficiently aware of interactions and interdependence between $\mathrm{OHC}$ and general care, and (b) related low prioritisation, and poor awareness of and attitude towards oral health (care) at macro-, meso- and micro level.

\section{ACKNOWLEDGEMENTS}

This research was part of the "Zorg Verbindt" project (project $\mathrm{nr}$ 203072), which is financially supported by EUREGIO-Rijn Waa (a consortium of INTERREG A Deutschland-Nederland, Province Nordrhein-Westfalen, Province Gelderland, and Province Limburg). The funders had no role in the study design, data collection and analysis, or preparation of the manuscript. The research team acknowledges all participants in the study.

\section{CONFLICT OF INTEREST}

The authors have no conflicts of interest to declare.

\section{AUTHORS' CONTRIBUTION}

The first author (DN), designed the study, conducted and analysed the interviews, lead the workshop and wrote the manuscript. The second author (AG) designed the study, conducted and analysed the interviews, co-presented the workshop and contributed to the manuscript. The third author (VL) co-designed the study, participated in analysis of the interviews' transcripts, participated in the workshop and contributed to the manuscript.

\section{ORCID}

Dominique Niesten (iD https://orcid.org/0000-0001-9463-0051

\section{REFERENCES}

1. Hoeksema AR. Oral Health in Frail Elderly. University of Groningen; 2016.

2. Marino R, Albala C, Sanchez H, Cea X, Fuentes A. Prevalence of diseases and conditions which impact on oral health and oral health self-care among older chilean. J Aging Health. 2015;27(1):3-16.

3. Petersen PE, Ogawa H. Promoting oral health and quality of life of older people - the need for public health action. Oral Health Prev Dent. 2018;16(2):113-124.

4. Manski R, Moeller J. Barriers to oral health across selected European countries and the USA. Int Dent J. 2017;67(3):133-138.

5. Harnagea $\mathrm{H}$, Lamothe L, Couturier $\mathrm{Y}$, et al. From theoretical concepts to policies and applied programmes: the landscape of integration of oral health in primary care. BMC Oral Health. 2018;18(1):23.

6. Harnagea $\mathrm{H}$, Couturier $\mathrm{Y}$, Shrivastava $\mathrm{R}$, et al. Barriers and facilitators in the integration of oral health into primary care: a scoping review. BMJ Open. 2017;7(9):e016078.

7. Atchison KA, Rozier RG, Weintraub JA. Integration of oral health and primary care: communication, coordination, and referral. NAM Perspectives. Discussion Paper. National Academy of Medicine, Washington, DC. 2018:1-12. https://doi.org/10.31478/201810e

8. Norwood CW, Maxey HL, Randolph C, Gano L, Kochhar K. Administrative challenges to the integration of oral health with primary care: a SWOT analysis of health care executives at federally qualified health centers. J Ambul Care Manage. 2017;40(3):204-213.

9. Briggs AM, Valentijn PP, Thiyagarajan JA, Araujo de Carvalho I. Elements of integrated care approaches for older people: a review of reviews. BMJ Open. 2018;8(4):e021194.

10. Gerritsen P, Cune M, van der Bilt A, Abbink J, de Putter C. Effects of integrated dental care on oral treatment needs in residents of nursing homes older than 70 years. Spec Care Dentist. 2015;35(3):132-137.

11. World Health Organization WHO. People-centred and Integrated Health Services: An Overview of the Evidence. World Health Organization; 2015. Contract No.: WHO/HIS/SDS/2015.7.

12. Rozenblum R, Lisby M, Hockey PM, et al. The patient satisfaction chasm: the gap between hospital management and frontline clinicians. BMJ Qual Saf. 2013;22(3):242-250.

13. Liabo K, Ingold A, Roberts H. Co-production with "vulnerable" groups: balancing protection and participation. Health Sci Rep. 2018;1(3):e19.

14. World Health Organization WHO. Integrated Care Models: An Overview. Programme HSD, Health DoHSaP; 2016.

15. Valentijn PP, Schepman SM, Opheij W, Bruijnzeels MA. Understanding integrated care: a comprehensive conceptual framework based on the integrative functions of primary care. Int $J$ Integr Care. 2013;13:e010.

16. EUREGIO. EUREGIO Rijn Waal. https://www.euregio.org/. Accessed January 12, 2020.

17. Patton MQ. Qualitative Research and Evaluation Methods (3rd edn). SAGE Publications; 2002. 
18. Jungk R, Müllert N. Future Workshops: How to Create Desirable Futures. Institute for Social Inventions; 1987.

19. Hsieh HF, Shannon SE. Three approaches to qualitative content analysis. Qual Health Res. 2005;15(9):1277-1288.

20. Grone O, Garcia-Barbero M. Integrated care: a position paper of the WHO European Office for Integrated Health Care Services. Int $J$ Integr Care. 2001;1:e21.

21. Krefting L. Rigor in qualitative research: the assessment of trustworthiness. Am J Occup Ther. 1991;1991(45):214-222.

22. VERENSO. Richtlijn mondzorg voor zorghafhankelijke cliënten in verpleeghuizen. 2007. https://www.verenso.nl/richtlijnen-en-praktijkvo ering/richtlijnendatabase/mondzorg. Accessed March 14, 2020.

23. Varpio L, Ajjawi R, Monrouxe LV, O'Brien BC, Rees CE. Shedding the cobra effect: problematising thematic emergence, triangulation, saturation and member checking. Med Educ. 2017;51(1):40-50.

24. Cunnion DT, Spiro A 3rd, Jones JA, et al. Pediatric oral health-related quality of life improvement after treatment of early childhood caries: a prospective multisite study. J Dent Child (Chic). 2010;77(1):4-11.

25. Valentijn PP, Vrijhoef HJ, Ruwaard D, Boesveld I, Arends RY, Bruijnzeels MA. Towards an international taxonomy of integrated primary care: a Delphi consensus approach. BMC Fam Pract. 2015;16:64.

26. Gostemeyer G, Baker SR, Schwendicke F. Barriers and facilitators for provision of oral health care in dependent older people: a systematic review. Clin Oral Investig. 2019;23(3):979-993.

27. Godin G, Belanger-Gravel A, Eccles M, Grimshaw J. Healthcare professionals' intentions and behaviours: a systematic review of studies based on social cognitive theories. Implement Sci. 2008;3:36.

28. Poulton BC, West MA. The determinants of effectiveness in primary health care teams. J Interprof Care. 1999;13(1):7-18.

29. Reeves S, Palaganas J, Zierler B. An updated synthesis of review evidence of interprofessional education. J Allied Health. 2017;46(1):56-61.

30. Vanobbergen J, Hollaar V, Sederel R, Vyt A. Interprofessional collaboration, the key to better oral healthcare for frail older people. Ned Tijdschr Tandheelkd. 2019;126(12):679-686.

31. Glassman P, Harrington M, Namakian M, Subar P. Interprofessional collaboration in improving oral health for special populations. Dent Clin North Am. 2016;60(4):843-855.

32. Zwarenstein M, Goldman J, Reeves S. Interprofessional collaboration: effects of practice-based interventions on professional practice and healthcare outcomes. Cochrane Database Syst Rev. 2009;(3):Cd000072.

33. Appelbaum NP, Lockeman KS, Orr S, Huff TA, Hogan CJ, Queen BA, Dow AW. Perceived influence of power distance, psychological safety, and team cohesion on team effectiveness. Journal of Interprofessional Care. 2020;34(1):20-26. http://dx.doi. org/10.1080/13561820.2019.1633290

34. Edmondson AC, Lei Z. Psychological safety: the history, renaissance, and future of an interpersonal construct. Annu Rev Organization Psychol Organization Behav. 2014;1(1):23-43.

35. Karam M, Brault I, Van Durme T, Macq J. Comparing interprofessional and interorganizational collaboration in healthcare: a systematic review of the qualitative research. Int J Nurs Stud. 2018;79:70-83.

36. World Health Organization WHO. Learning Together to Work Together for Health. World Health Organization; 1988.

37. World Health Organization WHO. Framework for Action on Interprofessional Education and Collaborative Practice. World Health Organization; 2010.
38. Jones JA, Snyder JJ, Gesko DS, Helgeson MJ. Integrated Medical-Dental Delivery Systems: models in a changing environment and their implications for dental education. J Dent Educ. 2017;81(9):eS21-eS29.

39. Atchison KA, Weintraub JA. integrating oral health and primary care in the changing health care landscape. N C Med J. 2017;78(6):406-409.

40. World Health Organization WHO. A Vision for Primary Health Care in the 21st Century. Towards Universal Health Coverage and the Sustainable Development Goals. World Health Organization; 2018.

41. Amerine C, Boyd L, Bowen DM, Neill K, Johnson T, Peterson T. Oral health champions in long-term care facilities-a pilot study. Spec Care Dentist. 2014;34(4):164-170.

42. National Health Service (UK). NHS Leadership Framework LF Quick Reference Guide Summary of Domains Elements and Stages. 2012.

43. Garrido Urrutia C, Romo Ormazabal F, Espinoza Santander I, Medics SD. Oral health practices and beliefs among caregivers of the dependent elderly. Gerodontology. 2012;29(2):e742-e747.

44. World Health Organization. New investments in global health workforce will create jobs and drive economic growth 2016. https://www.who.int/news-room/detail/20-09-2016-un-commi ssion-new-investments-in-global-health-workforce-will-creat e-jobs-and-drive-economic-growth. Accessed January 20, 2020.

45. Lee LS, O'Connor Grochowski C, Valiga TM, von Isenburg M, McNeill DB. Building Social Capital To Foster Interprofessional Education: The Interprofessional Educator Academy. Acad Med. 2019;11:1685-1690.

46. Kossioni AE, Hajto-Bryk J, Maggi S, et al. An Expert Opinion From the European College of Gerodontology and the European Geriatric Medicine Society: European Policy Recommendations on Oral Health in Older Adults. J Am Geriatr Soc. 2018;66(3):609-613.

47. Zorgwijzer. Kosten zorgverzekeringswet zvw met 15 miljard omhoog. 2020. https://www.zorgwijzer.nl/zorgverzekering-2020/ kosten-zorgverzekering swet-zvw-met-15-miljard-omhoog. Accessed March 23, 2020.

48. Brocklehurst P, Price J, Glenny AM, et al. The effect of different methods of remuneration on the behaviour of primary care dentists. Cochrane database Syst Rev. 2013;11:Cd009853.

49. Listl S, Chalkley M. Provider payment bares teeth: dentist reimbursement and the use of check-up examinations. Soc Sci Med. 2014;111:110-116.

50. McDonald R, Cheraghi-Sohi S, Sanders C, Tickle M. Changes to financial incentives in English dentistry 2006-2009: a qualitative study. Community Dent Oral Epidemiol. 2012;40(5):468-473.

51. Whittaker W, Birch S. Provider incentives and access to dental care: evaluating NHS reforms in England. Soc Sci Med. 2012;75(12):2515-2521.

52. Valentijn PP, Biermann C, Bruijnzeels MA. Value-based integrated (renal) care: setting a development agenda for research and implementation strategies. BMC Health Serv Res. 2016;16:330.

How to cite this article: Niesten D, Gerritsen AE, Leve V. Barriers and facilitators to integrate oral health care for older adults in general (basic) care in East Netherlands. Part 1: Normative integration. Gerodontology. 2021;38:154-165. https://doi.org/10.1111/ger.12507 